\title{
CREATING WAVE-FOCUSING MATERIALS
}

\author{
A.G. Ramm \\ Department of Mathematics \\ Kansas State University, \\ Manhattan, KS 66506-2602, USA \\ ramm@math.ksu.edu \\ fax 785-532-0546, tel. 785-532-0580
}

\begin{abstract}
Basic ideas for creating wave-focusing materials by injecting small particles in a given material are described.

The number of small particles to be injected around any point is calculated. Inverse scattering problem with fixed wavenumber and fixed incident direction of the plane acoustic wave is formulated and solved.
\end{abstract}

\section{INTRODUCTION}

This paper contains the results presented at the author's plenary talk at the IPDO2007 symposium on inverse problems, design and optimization. A method for creating materials with a desired refraction coefficient is given. This coefficient, in particular, may be chosen so that the new material has a desired wave-focusing property (see also [1]-[12]).

We want to investigate the following problem. Let $D$ be a bounded domain filled with a material whose properties are known, for example, a homogeneous material with known speed propagation of sound waves. Can one inject into $D$ small particles in such a way that the resulting new material would have some desirable wave-focusing properties? For example, is it possible to create in this way a material that scatters an incident plane wave in a desired solid angle?

There is a large engineering and physical literature on creating "smart" materials. Photonic crystals, quantum dots, coating, are some key words. However, there seems to be no prior work which deals with the question posed above.

In this paper this question is studied rigorously: the "smallness" of the particles is specified, the number of these particles

MSC: 35J05, 35J10, 35R30, 74J25, 81U40

PACS: 03.04.Kf

Key words: wave focusing, "smart" materials, inverse scattering, small particles around the point $x$ is specified, the role of the shapes of these particles is explained, and the notion of wave-focusing is made precise.

The basic results of this paper are:

1) It is proved that the injection of a suitable number of small, acoustically soft particles in a given bounded region, filled by some material with known properties, allows one to create a new material such that its scattering amplitude is arbitrarily close to a given scattering amplitude.

2) A method is given to calculate the density $N(x)$ of small particles, to be injected in a unit volume around a point $x \in D$, in order that the new material has the scattering amplitude close to the desired scattering amplitude.

3) For the first time the problem of finding a compactly supported potential $q(x)$ which generates the scattering amplitude $A(\beta):=$ $A(\beta, \alpha, k)$, approximating an arbitrary fixed given function $f(\beta) \in L^{2}\left(S^{2}\right)$ with any desired accuracy is formulated and solved. Here the wavenumber $k>0$ and the incident direction $\alpha \in S^{2}$ are fixed, $S^{2}$ is the unit sphere in $R^{3}$.

In Section 2 detailed statements of the problems are given. In Section 3 the inverse scattering problem with fixed wavenumber $k>0$ and fixed incident direction $\alpha \in S^{2}$ is discussed.

In Section 4 the ill-posedness of the above problems is discussed.

For engineers the paper gives a "recipe" for creating materials with a desired refraction coefficient. No such receipes were given earlier, to the author's knowledge, although there were many papers (see book [13] and references therein) in which an effect of embedding small spheres or ellipsoids into a homogeneous material on the effective dielectric and magnetic properties of the material was discussed, and the new material was a homogenized material. Homogenization in 
the literature was studied mostly for elliptic positive-definite operators, while we study wave propagation and the corresponding operator is not positive-definite.

Another principal difference between this paper and the earlier published results consists in the statement of the problem: we are not considering the distribution of small particles, embedded into the material, as uniform or random, but solve a design problem of creating materials with a desired refraction coefficient.

Moreover, we give (see also [10]-[12]) a precise recipe for creating such material for practically arbitrary refraction coefficient.

The two technological (engineering) problems to be solved for this recipe to be practically implemented, can be formulated precisely as well:

a) How does one practically embed in a given material many small particles given the number of the particles per unit volume around every point of the original material?

b) How does one prepare practically small particles with a desired boundary impedance?

In this paper we consider small particles with the Dirichlet boundary condition, corresponding to acoustically soft particles. Problem b) will arise if one considers the small particles on the boundary of which an impedance boundary condition is imposed. Varying the boundary impedance as a function of positions of particles, one can create refraction coefficients with the desired absorption properties. This was discussed in more detail in [12].

\section{STATEMENT OF THE PROBLEM AND SOME RESULTS}

Consider a bounded domain $D \subset \mathbb{R}^{3}$ with a smooth boundary $S$. The scattering of a plane wave on this domain is described by the equations

$$
\begin{aligned}
& {\left[\nabla^{2}+k^{2} n_{0}^{2}(x)\right] u=0 \text { in } D, k=\text { const }>0,} \\
& u=e^{i k \alpha \cdot x}+A_{0}(\beta, \alpha, k) \frac{e^{i k r}}{r}+o\left(\frac{1}{r}\right), \\
& r:=|x| \rightarrow \infty, \quad \beta:=\frac{x}{r} .
\end{aligned}
$$

Here $\alpha \in S^{2}$ is given, $A_{0}(\beta, \alpha, k)$ is the scattering amplitude, $k>0$ is fixed throughout the paper, $n_{0}^{2}(x)>0$ is a given function, the refraction coefficient, $n_{0}^{2}(x)=1$ in $D^{\prime}:=R^{3} \backslash D, n_{0}^{2}(x)$ is piecewise-continuous. The function $n_{0}^{2}(x)$ describes the material properties of the region $D$. Problem (1)-(2) has a unique solution $u \in H_{l o c}^{2}\left(R^{3}\right)$, where
$H_{l o c}^{2}\left(R^{3}\right)$ is the Sobolev space. Equation (1) can be written as a Schrödinger equation

$\left[\nabla^{2}+k^{2}-q_{0}(x)\right] u=0, \quad q_{0}(x):=k^{2}\left[1-n_{0}^{2}(x)\right]$,

$q_{0}=0$ in $D^{\prime}$. Suppose that $M$ small acoustically soft particles (bodies) $D_{m}$ are injected into domain $D$. Smallness means that $k a<<1$, where $a$ is the characteristic size of the small bodies. One may define $a:=\frac{1}{2} \max _{1 \leq m \leq M} \operatorname{diam} D_{m}$. Assume that the boundaries $S_{m}$ of $D_{m}$ are uniformly Lipschitz, i.e., the Lipschitz constant does not depend on $m$. Acoustically soft means that $\left.u\right|_{S_{m}}=0$, where $u$ can be interpreted as acoustic pressure.

The scattering problem in the new region can be formulated as follows:

$$
\begin{gathered}
{\left[\nabla^{2}+k^{2} n_{0}^{2}(x)\right] \mathcal{U}=0 \text { in } R^{3} \backslash \bigcup_{m=1}^{M} D_{m},} \\
\left.\mathcal{U}\right|_{S_{m}}=0, \quad 1 \leq m \leq M, \\
\mathcal{U}=u+\mathcal{A}_{M}(\beta, \alpha, k) \frac{e^{i k r}}{r}+o\left(\frac{1}{r}\right), \\
r \rightarrow \infty, \frac{x}{r}=\beta,
\end{gathered}
$$

where $u$ solves (1)-(2). One can replace (5) by the following:

$$
\begin{gathered}
\mathcal{U}=e^{i k \alpha \cdot x}+A(\beta, \alpha, k) \frac{e^{i k r}}{r}+o\left(\frac{1}{r}\right) \\
r \rightarrow \infty, \quad \frac{x}{r}=\beta .
\end{gathered}
$$

From (2), (5) and (6) one gets $A=A_{0}+\mathcal{A}_{M}$.

Since $n_{0}^{2}(x)$ is known, $A_{0}$ is known.

Problem (3)-(5) has a unique solution.

Problem 1 is to show that one can distribute sufficiently large number $M$ of small particles in $D$ in such a way that $A(\beta, \alpha):=$ $A(\beta, \alpha, k), k>0$ is fixed, will approximate in $L^{2}\left(S^{2}\right)$ an arbitrary fixed scattering amplitude $f(\beta, \alpha)$ with any desired accuracy. We consider also the following problem.

Problem 2: Can one distribute small particles in $D$ so that the resulting new material would have the scattering amplitude $A(\beta)$ ( $k>0$ and $\alpha \in S^{2}$ are both now fixed), which approximates in $L^{2}\left(S^{2}\right)$ an arbitrary given function $f(\beta) \in L^{2}\left(S^{2}\right)$ with any desired accuracy?

If $A(\beta, \alpha):=A(\beta, \alpha, k)(k>0$ is fixed $)$ is a scattering amplitude, known for all $\beta, \alpha \in S^{2}$, then one can find the unique, corresponding to $A(\beta, \alpha)$, potential $q(x)$ by the Ramm's method [1], [2]. This method gives a stable approximation of $q$ even in 
the case when noisy data $A_{\delta}(\beta, \alpha)$ are given, $\sup _{\beta, \alpha \in S^{2}} \mid A(\beta, \alpha)-A_{\delta}(\beta, \alpha \mid<\delta$.

If $q(x)$ is found from $A(\beta, \alpha)$, then we define $p(x):=q-q_{0}(x)$ and prove that $p(x)=N(x) C_{0}$, where $N(x)$ is the number of small particles per unit volume around a point $x$, i. e., the spatial density of the number of the particles, and $C_{0}$ is the electrical capacitance of a small conductor of the same shape as the particle. Here we assume that all the small particles are identical, but this assumption can be dropped (see [3], [4] ).

Let us summarize: If one injects small particles with the spatial density $N(x)=$ $\frac{p(x)}{C_{0}}$, where $p(x):=q(x)-q_{0}(x)$, then the resulting new material will have practically the desired scattering amplitude $A(\beta, \alpha)$, corresponding to a potential $q(x)$.

This gives a solution to Problem 1. Note that the scattering amplitude $A(\beta, \alpha)$, $\forall \beta, \alpha \in S^{2}$, corresponding to a real-valued potential $q \in L^{2}(D)$, determines $q$ uniquely. Since $q_{0}(x)$ is known, the function

$$
N(x)=\frac{q(x)-q_{0}(x)}{C_{0}}
$$

gives an approximate solution to Problem 1. This solution is not exact because we did not pass to the limit

$$
M \rightarrow \infty, \quad k a \rightarrow 0, \quad \frac{a}{d} \rightarrow 0,
$$

but took just sufficiently small identical particles with $C_{0}$ being electrical capacitance of the perfect conductor of the same shape as a single particle.

The number of small particles per unit volume is $O\left(\frac{1}{d^{3}}\right)$. Their volume per unit volume of the original medium is $O\left(\frac{a^{3}}{d^{3}}\right)$. This quantity tends to zero as $\frac{a}{d} \rightarrow 0$. The capacitance per unit volume is $O\left(\frac{a}{d^{3}}\right)$. Thus, the limit of the ratio $\frac{a}{d^{3}}$ is finite and nonzero, while the limit of the relative volume of the injected small particles is zero because $\frac{a^{3}}{d^{3}}$ tends to zero.

\section{INVERSE SCATTERING WITH FIXED $k$ AND $\alpha$}

Our solution to Problem 2 is based on the idea used in Section 2 in solving Problem 1. Given $f(\beta)$, we find $q(x) \in L^{2}(D)$, such that the corresponding to $q$ scattering amplitude $A_{q}(\beta)\left(k>0\right.$ and $\alpha \in S^{2}$ are fixed) approximates $f(\beta)$ with a desired accuracy: $\left\|f(\beta)-A_{q}(\beta)\right\|_{L^{2}\left(D^{2}\right)}<\varepsilon$, where $\varepsilon>0$ is an a priori given small number. If such a $q$ is found, then $N(x)=\frac{q(x)-q_{0}(x)}{C_{0}}$ as in Section 2. The principally novel problem is finding $q$ from $f(\beta)$ and $\varepsilon$. This problem has many solutions, as we prove. However, a priori it is not at all clear if this problem has a solution. Let us outline our solution to this problem. First, recall the well-known exact formula for the scattering amplitude:

$$
A_{q}(\beta)=-\frac{1}{4 \pi} \int_{D} e^{-i k \beta \cdot x} q(x) u(x) d x,
$$

where $u(x)$ is the scattering solution,

$$
\begin{gathered}
{\left[\nabla^{2}+k^{2}-q(x)\right] u=0 \text { in } R^{3},} \\
u=u_{0}+A_{q}(\beta) \frac{e^{i k r}}{r}+o\left(\frac{1}{r}\right), \quad r \rightarrow \infty,
\end{gathered}
$$

$\beta=\frac{x}{r}, u_{0}:=e^{i k \alpha \cdot x}, \alpha \in S^{2}$ and $k>0$ are fixed, $u(x)=u(x, \alpha, k)$. Denote $h(x)=$ $q(x) u(x)$. Then

$$
A_{q}=-\frac{1}{4 \pi} \int_{D} e^{-i k \beta \cdot x} h(x) d x,
$$

where $A_{q}$ is the scattering amplitude, corresponding to the potential $q$. Given $f(\beta)$ and $\varepsilon>0$, however small, one can find (many) $h$ such that

$$
\left\|f(\beta)+\frac{1}{4 \pi} \int_{D} e^{-i k \beta \cdot x} h(x) d x\right\|_{L^{2}\left(S^{2}\right)}<\varepsilon .
$$

The function $h$ can be found, for example, as a linear combination $h_{n}=\sum_{j=1}^{n} c_{j} \varphi_{j}(x)$, where $\left\{\varphi_{j}\right\}$ is a basis of $L^{2}(D)$. If $n$ is sufficiently large and $c_{j}$ are found from the minimization problem

$\left\|f+\sum_{j=1}^{n} \frac{c_{j}}{4 \pi} \int_{D} e^{-i k \beta \cdot x} \varphi_{j}(x) d x\right\|_{L^{2}\left(S^{2}\right)}=\min$,

then (7) holds. In [4] another, analytical, solution to $(7)$ is given.

If $h=h_{\varepsilon}(x)$ is found, then $q(x)$ can be found from the nonlinear equation $h=q u$. This equation for $q$ is nonlinear because the scattering solution $u=u(x ; q)$ depends nonlinearly on $q$. One has

$$
u(x)=u_{0}(x)-\int_{D} g(x, y) q(y) u(y) d y,
$$

where $u_{0}=e^{i k \alpha \cdot x}$ and $g:=\frac{e^{i k|x-y|}}{4 \pi|x-y|}$. Let

$$
q(x):=\frac{h(x)}{u_{0}(x)-\int_{D} g(x, y) h(y) d y} .
$$

If the right side of $(9)$ is an $L^{2}(D)$ function, then (9) solves our inverse scattering problem. Indeed, define

$$
u(x):=u_{0}(x)-\int_{D} g(x, y) h(y) d y .
$$


This $u$ solves (8) with $q$ defined in (9). The scattering amplitude

$$
A_{q}(\beta)=-\frac{1}{4 \pi} \int_{D} e^{-i k \beta \cdot x} h(x) d x .
$$

By (7) one has

$$
\left\|f(\beta)-A_{q}(\beta)\right\|_{L^{2}\left(S^{2}\right)}<\varepsilon .
$$

So, Problem 2 is solved if (9) defines an $L^{2}(D)$ function. This, for example, is the case if

$$
\inf _{x \in D}\left|u_{0}(x)-\int_{D} g(x, y) h(y) d y\right|>0 .
$$

If formula (9) defines a non-integrable function due to possible zero sets of the function

$$
\psi(x):=u_{0}-\int_{D} g(x, y) h(y) d y,
$$

then, as we prove, a suitable small perturbation $h_{\delta}$ of $h$ in $L^{2}(D)$-norm will lead to a function

$$
q_{\delta}:=\frac{h_{\delta}}{\psi_{\delta}} \in L^{2}(D)
$$

where

$$
\psi_{\delta}=u_{0}-\int_{D} g(x, y) h_{\delta}(y) d y,
$$

and $\left\|h-h_{\delta}\right\|_{L^{2}(D)}<\delta$. Since the function $-\frac{1}{4 \pi} \int_{D} e^{-i k \beta \cdot x} h_{\delta}(x) d x$ differs a little from the function $-\frac{1}{4 \pi} \int_{D} e^{-i k \beta \cdot x} h(x) d x$, condition (7) is satisfied if $h$ is replaced by $h_{\delta}$ and $\varepsilon$ by, for example, $2 \varepsilon$. Therefore

$$
N_{\delta}(x):=\frac{q_{\delta}(x)-q_{0}(x)}{C_{0}}
$$

solves Problem 2 in the case when (9) is not an $L^{2}(D)$-function.

Let us explain how to choose $h_{\delta}$. First, without loss of generality one can assume $h$ to be analytic in $\bar{D}=D \bigcup S$, because analytic functions (even polynomials if $D$ is bounded) are dense in $L^{2}(D)$. If $h$ is analytic, so is $\psi(x)$ in $D$. Therefore the null set of $\psi$,

$$
\mathcal{N}:=\{x: \psi(x)=0, x \in D\},
$$

is generically a line defined by two equations

$$
\psi_{1}:=\operatorname{Re} \psi=0, \quad \psi_{2}:=\operatorname{Im} \psi=0 .
$$

Let

$$
\mathcal{N}_{\delta}:=\{x:|\psi|<\delta, x \in D\},
$$

and $D_{\delta}:=D \backslash \mathcal{N}_{\delta}$. Generically $|\nabla \psi| \geq c>$ 0 on $\mathcal{N}$ and, by continuity, this inequality holds in $\mathcal{N}_{\delta}$ (possibly with a different $c>0$ ).
Small perturbation of $h$ leads to these generic assumptions.

Define

$$
\begin{aligned}
h_{\delta} & =\left\{\begin{array}{l}
h \text { in } D_{\delta}, \\
0 \text { in } \mathcal{N}_{\delta},
\end{array}\right. \\
q_{\delta} & := \begin{cases}\frac{h_{\delta}(x)}{u_{0}-\int_{D} g(x, y) h_{\delta}(y) d y} & \text { in } D_{\delta}, \\
0 & \text { in } \mathcal{N}_{\delta} .\end{cases}
\end{aligned}
$$

Then $q_{\delta} \in L^{2}(D)$ and

$$
N_{\delta}(x):=\frac{q_{\delta}(x)-q_{0}(x)}{C_{0}}
$$

solves Problem 2.

Let us check that $q_{\delta} \in L^{2}(D)$. We prove more: $q_{\delta} \in L^{\infty}(D)$. It is sufficient to check that

$$
\begin{aligned}
& \inf _{x \in D_{\delta}}\left|\psi_{\delta}(x)\right|= \\
& \inf _{x \in D_{\delta}}\left|u_{0}(x)-\int_{D_{\delta}} g(x, y) h(y) d y\right| \geq c \delta>0,
\end{aligned}
$$

because $q_{\delta}=0$ in $\mathcal{N}_{\delta}$ by the definition.

Choose the origin on $\mathcal{N}$ and make a change of variables

$$
s_{1}=\psi_{1}(x), s_{2}=\psi_{2}(x), s_{3}=x_{3} .
$$

The Jacobian of this transformation of variables is non-singular because $\nabla \psi_{1}$ and $\nabla \psi_{2}$ are linearly independent on $\mathcal{N}$ and in $\mathcal{N}_{\delta}$. We have

$$
\max _{x \in \mathcal{N}_{\delta}}\left(|J|+\left|J^{-1}\right|\right) \leq c,
$$

$c>0$ stands for a generic constant independent of $\delta$. Let us check that

$$
\left|\psi_{\delta}(x)\right| \geq c \delta \quad \text { in } \quad D_{\delta} .
$$

We have

$$
\left|\psi_{\delta}(x)\right| \geq|\psi(x)|-I(\delta),
$$

where

$$
I(\delta)=\int_{\mathcal{N}_{\delta}}|g(x, y) h(y)| d y .
$$

If $x \in D_{\delta}$, then $|\psi| \geq \delta$, and

$$
\left|\psi_{\delta}(x)\right| \geq \delta-I(\delta) .
$$

Moreover, $\max _{y \in D}|h| \leq M$ and, using the new variables $(12)$, one gets

$$
I(\delta) \leq c \int_{\rho \leq \delta} d \rho \rho \int_{0}^{1} \frac{d s_{3}}{\sqrt{s_{3}^{2}+\rho^{2}}},
$$

because the region $\mathcal{N}_{\delta}$ can be described by the inequalities

$$
\rho^{2}=s_{1}^{2}+s_{2}^{2} \leq \delta^{2}, \quad 0 \leq s_{3} \leq 1,
$$


and we have used the estimate $\left|J^{-1}\right| \leq c$ in $\mathcal{N}_{\delta}$. Integral

$$
I(\delta)=O\left(\delta^{2}|\ln \delta|\right) \quad \text { as } \quad \delta \rightarrow 0 .
$$

Thus

$$
\left|\psi_{\delta}\right| \geq \delta-O\left(\delta^{2}|\ln \delta|\right) \geq c \delta
$$

with some constant $c \in(0,1)$. This justifies our method for solving Problem 2 in the case when formula (9) does not yield $q \in L^{2}(D)$.

\section{ILL-POSEDNESS OF PROBLEMS 1 AND 2}

Both Problems 1 and 2 are ill-posed. Since the ill-posedness of Problem 1 has been discussed in great detail in [1], [2], we discuss only the ill-posedness of Problem 2.

In Problem 2 one has to find $h$, given $f$ and $\varepsilon>0$, so that (7) holds. This is an ill-posed problem, similar to solving the first kind integral equation

$$
B h:=-\frac{1}{4 \pi} \int_{D} e^{-i k \beta \cdot x} h(x) d x=f(\beta)
$$

for $h$. If this equation is solvable for a given $f$, it may be not solvable if $f$ is replaced by a slightly perturbed function $f_{\delta}$. If $\varepsilon>0$ in (7) is small and $f$ is not in the range of $B$, then $\|h\|_{L^{2}(D)}$ is large. This leads to large maximal values of the corresponding $q$. Therefore any numerical method for solving Problem 2 has to use a regularization procedure. In [5] one can find some numerical results related to Problem 2 and a description of the regularization procedure which was used.

\section{REFERENCES}

[1] A. G. Ramm, Stability of solutions to inverse scattering problems with fixedenergy data, Milan Journ of Math., 70, (2002), 97-161.

[2] Ramm, A. G. , Inverse problems, Springer, New York, 2005.

[3] A. G. Ramm, Completeness of the set of scattering amplitudes, Phys. Lett. A, $360, \mathrm{~N} 1,(2006), 22-25$.

[4] A. G. Ramm, Distribution of particles which produces a "smart" material, Journ. Stat. Phys., 127, N5, (2007), 914934.

[5] A. G. Ramm and S. Gutman, Computational method for acoustic wave focusing, Intern. Journ. Comp. Sci. and Math., 1, N1, (2007), 1-15.
[6] A. G. Ramm, Scattering by many small bodies and applications to condensed matter physics, Europ. Phys. Lett., 80, (2007), 44001.

[7] A. G. Ramm, Materials with the desired refraction coefficients can be made by embedding small particles, Phys. Lett. A, 370, 5-6, (2007), 522-527.

[8] A. G. Ramm, Many-body wave scattering by small bodies and applications, J. Math. Phys., 48, N10, (2007), 103511.

[9] A. G. Ramm, Distribution of particles which produces a desired radiation pattern, Physica B, 394, N2, (2007), 253255 .

[10] A. G. Ramm, A recipe for making materials with negative refraction in acoustics, Phys. Lett. A, 372/13, (2008), 2319-2321.

[11] A. G. Ramm, Scattering by many small bodies and applications to condensed matter physics, Europ. Phys. Lett., 80, (2007), 44001.

[12] A. G. Ramm, Wave scattering by many small particles embedded in a medium, Phys. Lett. A, 372/17, (2008), 30643070 .

[13] G. Milton, The theory of composites, Cambridge University Press, Cambridge, 2002. 